\title{
A Parallel Model for Predicting the Horizontal Dynamic Behavior of Helical Wire Rope Isolators
}

\author{
[ Nicolò Vaiana, Mariacristina Spizzuoco, Giorgio Serino ]
}

\begin{abstract}
In this paper, a one-dimensional (1d) parallel model able to simulate the horizontal dynamic behavior of Helical Wire Rope Isolators (HWRIs) in both Roll and Shear directions is proposed. The experimental tests performed on a HWRI, manufactured by Powerflex S.r.l. (Limatola, Italy), are first presented in order to describe the dynamic response of the tested metal device at different displacement amplitudes without and under the effect of the vertical load. The parallel modeling concept has been applied to discretize an exponential function proposed to reproduce the variation of the horizontal tangent stiffness at both small and large displacements, thus allowing to simulate the dynamic behavior of such devices by putting in parallel a purely linear elastic element, and a sufficiently high number of simple elastic-perfectly plastic elements and linear elastic gap elements. The mathematical model, requiring the evaluation of only five parameters from experimental data, has been validated by comparing the experimental force-displacement hysteresis loops obtained during cyclic tests with those predicted numerically. Good agreement between the simulated and experimental results shows that the proposed model can be an effective numerical tool to predict the horizontal force-displacement relationship of HWRIs. The presented parallel model, which allows to capture the feature of the smooth transition of the hysteresis loops from the small to large horizontal displacements ranges by adopting only one set of five parameters determined from experimental loops with the largest amplitude, is at the same time sufficiently versatile to be easily implemented in existing nonlinear finite element computer programs. Furthermore, compared to differential equation models, such as the widely used Wen model, the proposed one allows to avoid the numerical solution of a first order nonlinear ordinary differential equation for each time step of a nonlinear time history analysis, thus reducing the computation effort.
\end{abstract}

Keywords - helical wire rope isolators, nonlinear dynamic analysis, parallel modeling, seismically base-isolated structures

\section{Introduction}

Wire rope isolators are metal devices which are widely used in the shock and vibration isolation of industrial and defense equipment, electronic systems, critical machinery and other sensitive equipment because of their good dry friction damping performance [1]. These metal devices, also adopted for the seismic protection of light but costly equipment [2] and high voltage ceramic circuit breakers [3], [4], [5], [6], [7], have been recently used as vertical isolation

Nicolò Vaiana, Mariacristina Spizzuoco, Giorgio Serino University of Naples Federico II

Department of Structures for Engineering and Architecture Italy bearings in the antiseismic basements developed specifically for the base isolation of the two statues known as "Bronzes of Riace", located at the Archaeological Museum of Reggio Calabria, Italy [8]. Shaking table tests performed at the Department of Structures for Engineering and Architecture of the University of Naples Federico II (Italy) on a scaled structure, seismically isolated using four curved surface sliders and four wire rope isolators, have shown that the latter can be strongly useful when the complete recentering of the base-isolated structure is required and when the displacements of the base isolation system have to be reduced [9].

In this paper, the dynamic experimental tests performed at the Department of Industrial Engineering of the University of Naples Federico II (Italy) on a Helical Wire Rope Isolator (HWRI), manufactured by Powerflex S.r.1 (Limatola, Italy), are first presented in order to identify the mechanical characteristics of the tested device in the two principal horizontal directions, namely Roll and Shear directions.

After the description of the experimental results, a onedimensional (1d) parallel model, able to simulate the horizontal dynamic behavior of HWRIs at both small and large displacements, is proposed. It adopts a small set of model parameters to be evaluated from experimental data and allows to avoid the numerical solution of a first order nonlinear differential equation, for each time step of a nonlinear dynamic analysis, required by differential equation models such as the widely used Wen model [10]. The basic idea of the proposed model, obtained by applying the parallel modeling concept to discretize an exponential function proposed by the authors to represent the variation of the horizontal tangent stiffness within the large displacements range, is to consider one purely linear elastic element and a sufficiently high number of nonlinear elasticperfectly plastic elements connected in parallel, all having the same deformation but each carrying a different force, and to calculate the total restoring force of the parallel assemblage by summing the forces acting on each element. In order to model the increasing tangent stiffness displayed at large displacements, linear elastic gap elements are connected in parallel to the above-described assemblage.

The mathematical model has been validated by comparing the experimental hysteresis loops obtained during cyclic tests with those predicted numerically.

\section{Horizontal dynamic behavior of Helical Wire Rope Isolators}

HWRIs are metal devices made of a wire rope, constructed by layering several strands around a central one, which is wound in the form of a helix and embedded into two drilled metal retainer bars. 
Proc. of the Fourth International Conference on Advances in Civil, Structural and Environmental Engineering - ACSEE 2016. Copyright $($ Institute of Research Engineers and Doctors. All rights reserved. ISBN: 978-1-63248-114-6 doi: 10.15224/ 978-1-63248-114-6-29

The selected HWRI, manufactured by Powerflex S.r.l (Limatola, Italy), has a rope of six strands, each having 25 steel wires, plus a central one consisting of 49 wires. The material of the cable is Stainless Steel Type 316 whereas the one of the two retainer bars is aluminum alloy.

Fig. 1 shows the geometrical characteristics of the tested HWRI and its two principal horizontal directions, namely Roll and Shear directions.
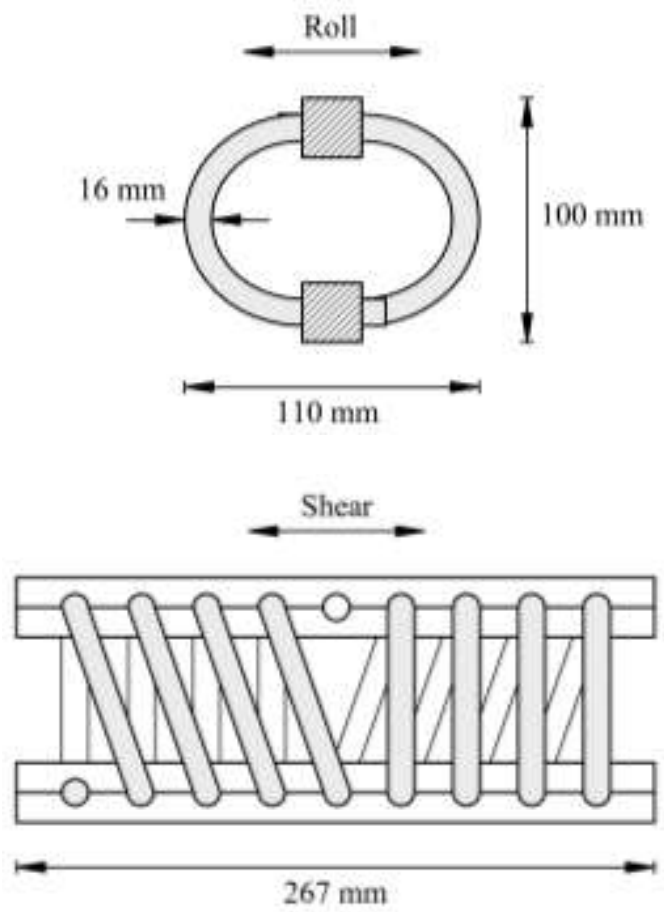

Figure 1. Geometrical characteristics and principal horizontal directions of the tested HWRI.

The selected HWRI has been tested in Roll and Shear directions by adopting a testing machine (Fig. 2), available at the Department of Industrial Engineering of the University of Naples Federico II (Italy), consisting of a horizontal hydraulic actuator, powered by a $75 \mathrm{~kW}$ AC electric motor, which allows to impose both load or displacement histories. The maximum horizontal force is 50 $\mathrm{kN}$, the maximum speed is $2.2 \mathrm{~m} / \mathrm{s}$, the maximum stroke is \pm $0.2 \mathrm{~m}$. A hydraulic jack allows to apply a vertical load up to $190 \mathrm{kN}$ during the tests [11].

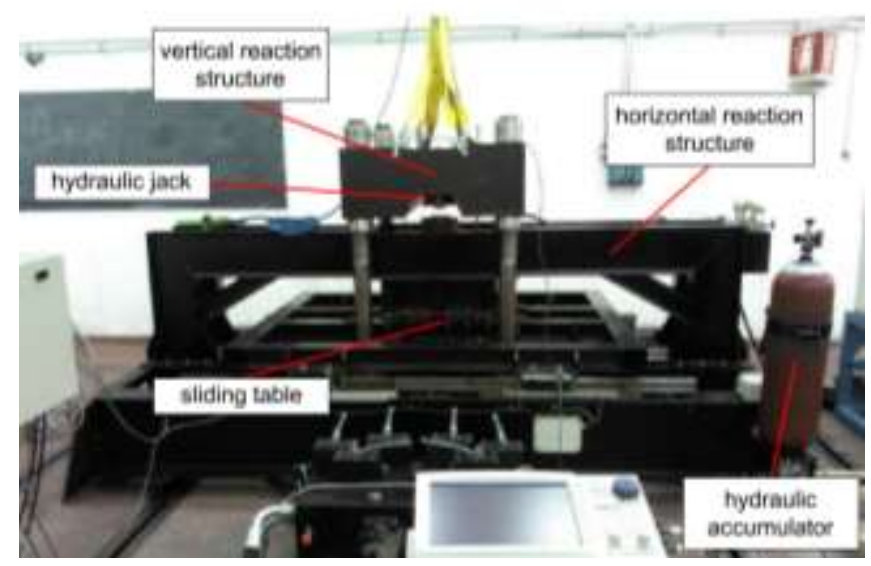

Figure 2. Testing Machine.
A sinusoidal displacement, consisting of five reversed cycles and having frequency $f=1 \mathrm{~Hz}$, was imposed for three different values of amplitude, that is, $A=0.0025 \mathrm{~m}, A=$ $0.005 \mathrm{~m}$, and $A=0.01 \mathrm{~m}$, without and under the effect of a vertical load $P_{v}=2 \mathrm{kN}$.

Fig. 3-5 show the symmetric force-displacement hysteresis loops obtained in Roll and Shear directions at small, relatively large, and large displacements, respectively. These experimental results reveal that the shape of the hysteresis loops changes according to the displacements range: at small displacements the hysteresis loops display a softening stiffness (Fig. 3) whereas at larger displacements the device exhibits a hardening stiffness (Fig. 4-5).

(a)

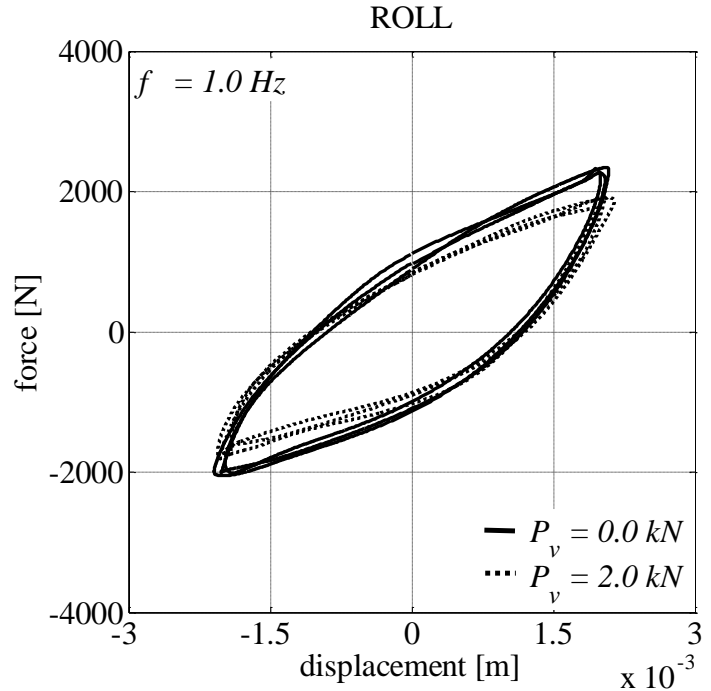

(b)

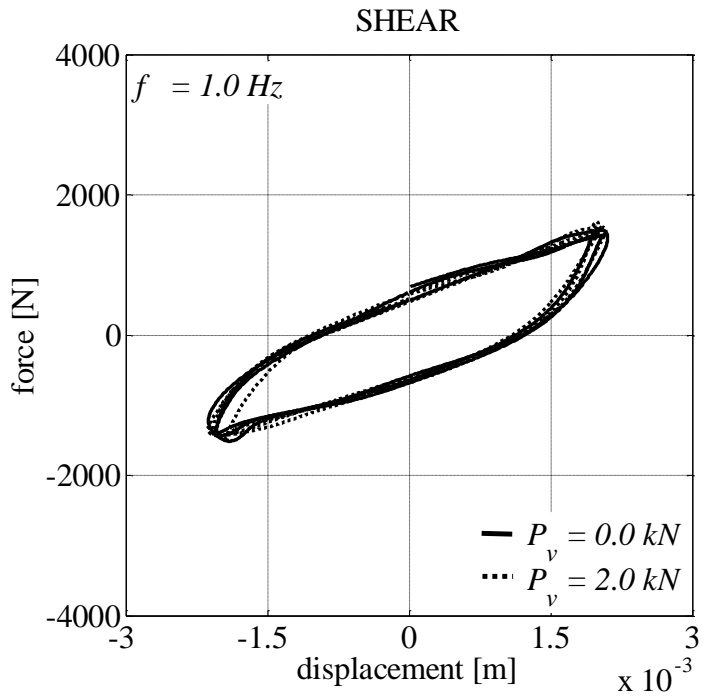

Figure 3. Force-displacement hysteresis loops at small displacements in (a) Roll and (b) Shear directions. 
Proc. of the Fourth International Conference on Advances in Civil, Structural and Environmental Engineering - ACSEE 2016.

(a)

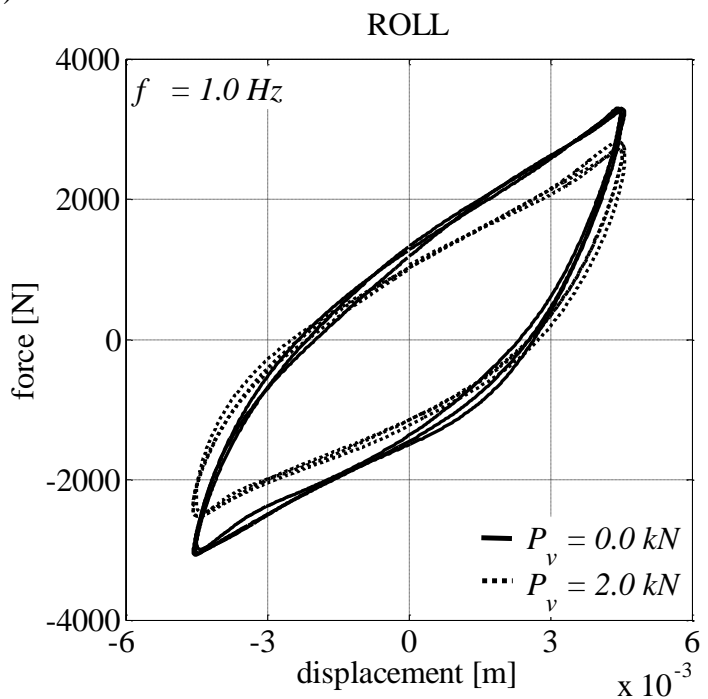

(b)

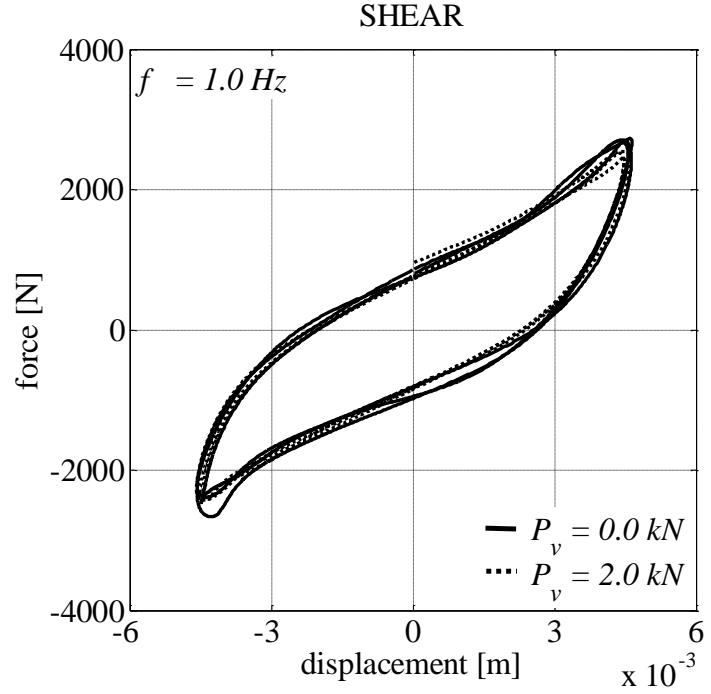

Figure 4. Force-displacement hysteresis loops at relatively large displacements in (a) Roll and (b) Shear directions.

(a)

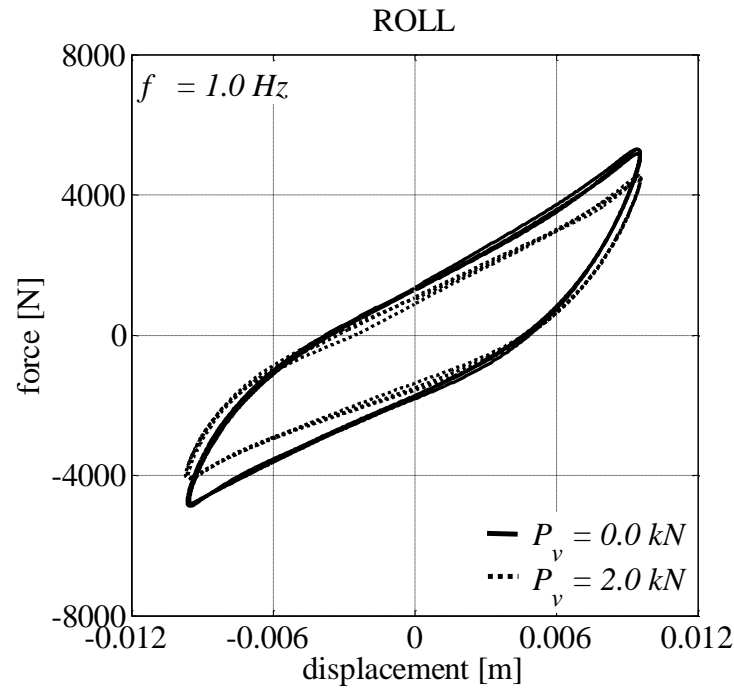

(b)

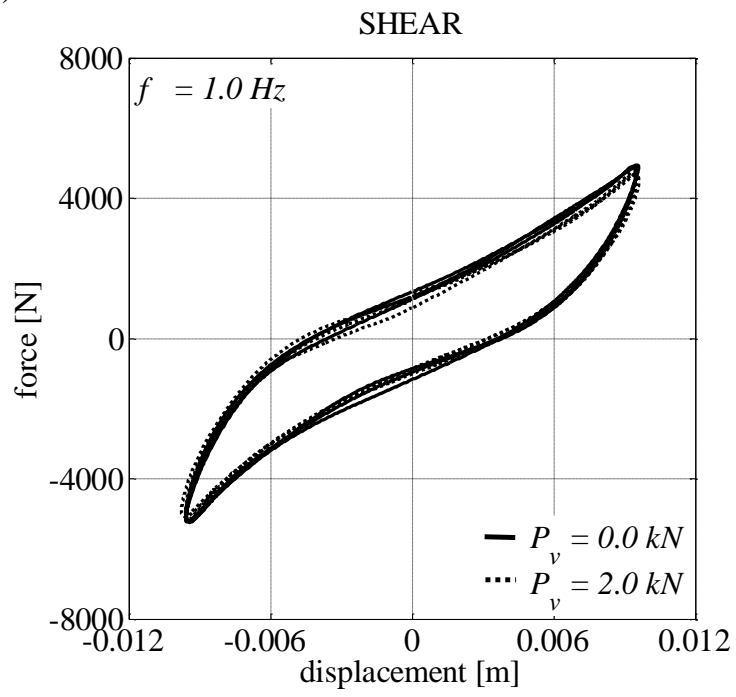

Figure 5. Force-displacement hysteresis loops at large displacements in (a) Roll and (b) Shear directions.

\section{Parallel modeling of Helical Wire Rope Isolators}

In this section, a 1d parallel model is proposed to reproduce the dynamic behavior of HWRIs at small and large displacements in both Roll and Shear directions.

According to the experimental results presented in Section II, the horizontal tangent stiffness exponentially decreases with increasing displacement within the small displacements range (Fig. 3). Thus, it can be expressed by the following two mathematical expressions, valid for a loading and an unloading curve, respectively:

$$
\begin{array}{ll}
k_{t 1}(u)=k_{\infty}+\left(k_{O}-k_{\infty}\right) e^{-c_{I}\left(u-u_{\min }\right)}, & (\dot{u}>0) \\
k_{t 1}(u)=k_{\infty}+\left(k_{O}-k_{\infty}\right) e^{-c_{I}\left(u_{\max }-u\right)}, & (\dot{u}<0)
\end{array}
$$

where $k_{0}$ and $k_{\infty}$ are the initial and the asymptotic values of the tangent stiffness within the small displacements range, $u_{\max }$ and $u_{\min }$ are the horizontal displacement values at the most recent point of unloading and loading, respectively, and $c_{1}$ is a parameter that defines the transition from $k_{0}$ to $k_{\infty}$. Equations (1) and (2) can be written in a more compacted form as follows:

$$
k_{t 1}(u)=k_{\infty}+a e^{-c_{1} u},
$$

valid for a generic loading or unloading curve obtained during cyclic testing on a single metal device, where $a=k_{0}-k_{\infty}$ and $u$ is evaluated starting from the latest point of unloading or reloading.

The increase of the tangent stiffness displayed at larger displacements (Fig. 4-5) can be obtained by summing the following mathematical expression to the previous one:

$$
k_{t 2}(u)=-c_{2}+c_{2} e^{\left[\operatorname{sign}(u) c_{3} u\right]},
$$


Proc. of the Fourth International Conference on Advances in Civil, Structural and Environmental Engineering - ACSEE 2016. Copyright (C) Institute of Research Engineers and Doctors. All rights reserved. ISBN: 978-1-63248-114-6 doi: 10.15224/ 978-1-63248-114-6-29

where $c_{2}$ and $c_{3}$ are two parameters of the proposed exponential function.

The continuously decreasing tangent stiffness function given by (3) can be approximated through a piecewise constant function with $N+1$ equally spaced decreasing values of $k_{t 1}(u)$, as shown in Fig. 6.

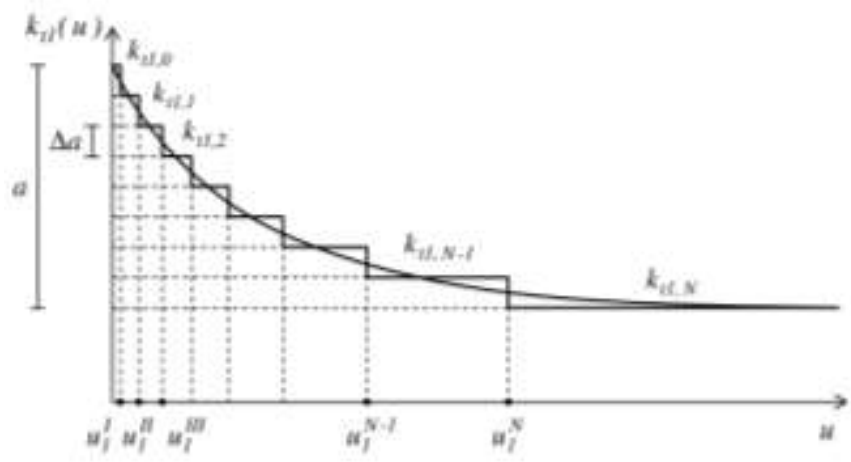

Figure 6. Continuous and discretized tangent stiffness function $k_{t 1}(u)$.

Assuming $\Delta a=a / N$, the discretized tangent stiffness values are:

$$
\begin{gathered}
k_{t 1,0}=k_{\infty}+a, \\
k_{t 1,1}=k_{\infty}+(N-1) \Delta a, \\
k_{t 1,2}=k_{\infty}+(N-2) \Delta a, \\
\ldots, \\
k_{t 1, N-1}=k_{\infty}+\Delta a, \\
k_{t 1, N}=k_{\infty},
\end{gathered}
$$

and the corresponding values of the horizontal displacements are obtained by solving (3) with respect to $u$ :

$$
u_{1, j}=-\frac{1}{c_{1}} \log \left(\frac{k_{t 1, j}-k_{\infty}}{a}\right) j=0,1, \ldots, N-1 .
$$

Thus, the expression of the tangent stiffness $k_{t 1}(u)$ can be approximated through the piecewise constant function given by:

$$
\begin{gathered}
u \leq u_{1}^{I} \rightarrow k_{t 1}(u)=k_{t 1,0}=k_{\infty}+a, \\
u_{1}^{I} \leq u<u_{1}^{I I} \rightarrow k_{t 1}(u)=k_{t 1,1}, \\
u_{1}^{I I} \leq u<u_{1}^{I I I} \rightarrow k_{t 1}(u)=k_{t 1,2}, \\
\quad \ldots, \\
u_{1}^{N-1} \leq u<u_{1}^{N} \rightarrow k_{t 1}(u)=k_{t 1, N-1}, \\
u \geq u_{1}^{N} \rightarrow k_{t 1}(u)=k_{t 1, N}=k_{\infty},
\end{gathered}
$$

where the limit displacement values $u_{1}^{i}$ are:

$$
u_{1}^{I}=\frac{u_{1,0}+u_{1,1}}{2}
$$

$$
\begin{gathered}
u_{1}^{I I}=\frac{u_{1,1}+u_{1,2}}{2}, \\
\ldots, \\
u_{1}^{N-1}=\frac{u_{1, N-2}+u_{1, N-1}}{2}, \\
u_{1}^{N}=\alpha u_{1, N-1},
\end{gathered}
$$

and $\alpha$ is an appropriate constant.

According to Masing's rule, the first loading curve, namely virgin curve, can be obtained from the generic loading curve from a similitude transformation of ratio 0.5 . This means that for a given $u$ on the virgin curve, where $u$ is computed starting from zero, the corresponding tangent stiffness must be equal to the one obtained from the generic loading curve for a value of $2 u$. Thus, in order to obtain the tangent stiffness for the virgin curve, it is just necessary to substitute $c_{1}$ with $2 c_{1}$ in (3):

$$
k_{t 1}(u)=k_{\infty}+a e^{-2 c_{1} u} .
$$

The continuously increasing tangent stiffness function given by (4) can be approximated through a piecewise constant function with $M+1$ equally spaced increasing values of $k_{t 2}(u)$, as shown in Fig. 7.

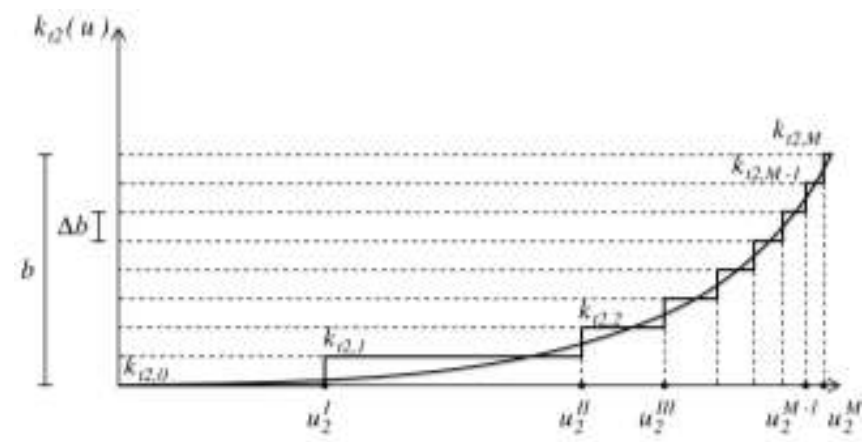

Figure 7. Continuous and discretized tangent stiffness function $k_{t 2}(u)$.

Assuming $\Delta b=b / M$, the discretized tangent stiffness values are:

$$
\begin{gathered}
k_{t 2,0}=0, \\
k_{t 2,1}=\Delta b, \\
k_{t 2,2}=2 \Delta b, \\
\ldots, \\
k_{t 2, M-1}=(M-1) \Delta b, \\
k_{t 2, M}=M \Delta b=b,
\end{gathered}
$$

and the corresponding values of the horizontal displacements are obtained by solving (4) with respect to $u$ :

$$
u_{2, j}=\frac{1}{c_{3}} \log \left(\frac{k_{t 2, j}+c_{2}}{c_{2}}\right) \quad j=0,1,2, \ldots, M .
$$


Proc. of the Fourth International Conference on Advances in Civil, Structural and Environmental Engineering - ACSEE 2016. Copyright (C) Institute of Research Engineers and Doctors. All rights reserved. ISBN: 978-1-63248-114-6 doi: 10.15224/ 978-1-63248-114-6-29

Thus, the expression of the tangent stiffness $k_{t 2}(u)$ can be approximated through the piecewise constant function given by:

$$
\begin{gathered}
u \leq u_{2}^{I} \rightarrow k_{t 2}(u)=k_{t 2,0}, \\
u_{2}^{I} \leq u<u_{2}^{I I} \rightarrow k_{t 2}(u)=k_{t 2,1}, \\
u_{2}^{I I} \leq u<u_{2}^{I I I} \rightarrow k_{t 2}(u)=k_{t 2,2}, \\
\ldots, \\
u_{2}^{M-1} \leq u<u_{2}^{M} \rightarrow k_{t 2}(u)=k_{t 2, M-1}, \\
u \geq u_{2}^{M} \rightarrow k_{t 2}(u)=k_{t 2, M}=b,
\end{gathered}
$$

where the limit displacement values $u_{2}^{i}$ are:

$$
\begin{gathered}
u_{2}^{I}=\frac{u_{2,0}+u_{2,1}}{2}, \\
u_{2}^{I I}=\frac{u_{2,1}+u_{2,2}}{2}, \\
u_{2}^{M-1}=\frac{u_{2, M-2}+u_{2, M-1}}{2}, \\
u_{2}^{M}=\frac{u_{2, M-1}+u_{2, M}}{2} .
\end{gathered}
$$

The above-described discretization of the two tangent stiffness functions, namely $k_{t 1}(u)$ and $k_{t 2}(u)$, allows to simulate the horizontal dynamic behavior of HWRIs by adopting a parallel assemblage made of one 1d elastic element having stiffness $k_{e l}=k_{\infty}, N$ 1d elastic-perfectly plastic elements, having stiffness $k_{e p, i}=\Delta a$ and yielding displacement $u_{1}^{i}$, for $i=I, I I, \ldots, N$, and $M 1 \mathrm{~d}$ linear elastic gap elements, having stiffness $k_{e g, i}=\Delta b$ and gap length equal to $u_{2}^{i}$, for $i=I, I I, \ldots, M$.

\section{Iv. Comparison between numerical and experimental results}

In order to show the validity of the proposed mathematical model, the results predicted numerically are compared to the experimental ones presented in Section II.

Fig. 8-10 give the comparisons of the experimental and parallel model results obtained in Roll direction, under the effect of a vertical load of $2 \mathrm{kN}$ and for a test frequency of 1 $\mathrm{Hz}$, at small (i.e., $A=0.0025 \mathrm{~m}$ ), relatively large (i.e., $A=$ $0.005 \mathrm{~m}$ ), and large (i.e., $A=0.01 \mathrm{~m}$ ) displacements, respectively. The results predicted adopting the proposed model have been obtained by using 50 elastic-perfectly plastic elements (i.e., $N=50$ ) and 50 linear elastic gap elements (i.e., $M=50$ ). It can be observed that the agreement between the experimental and numerical responses is very satisfactory.

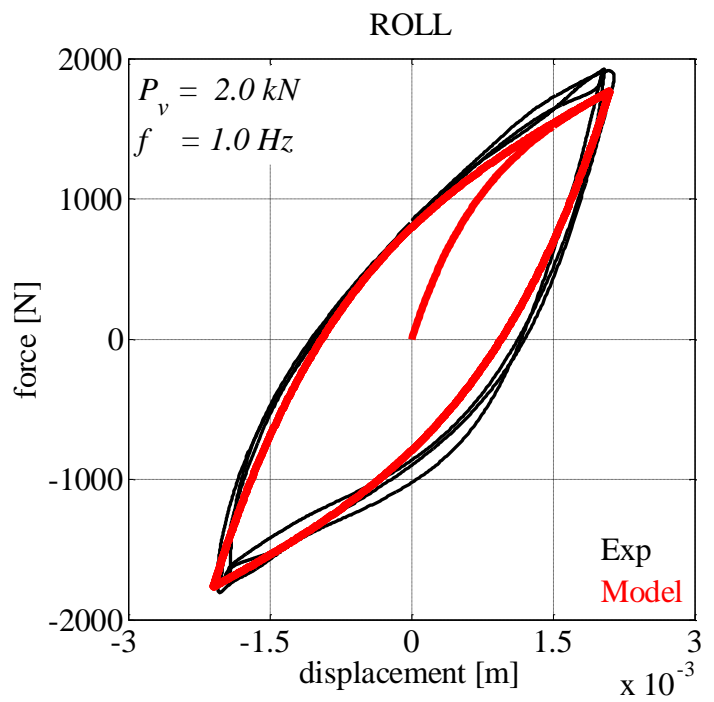

Figure 8. Experimental and parallel model loops for $A=0.0025 \mathrm{~m}$.

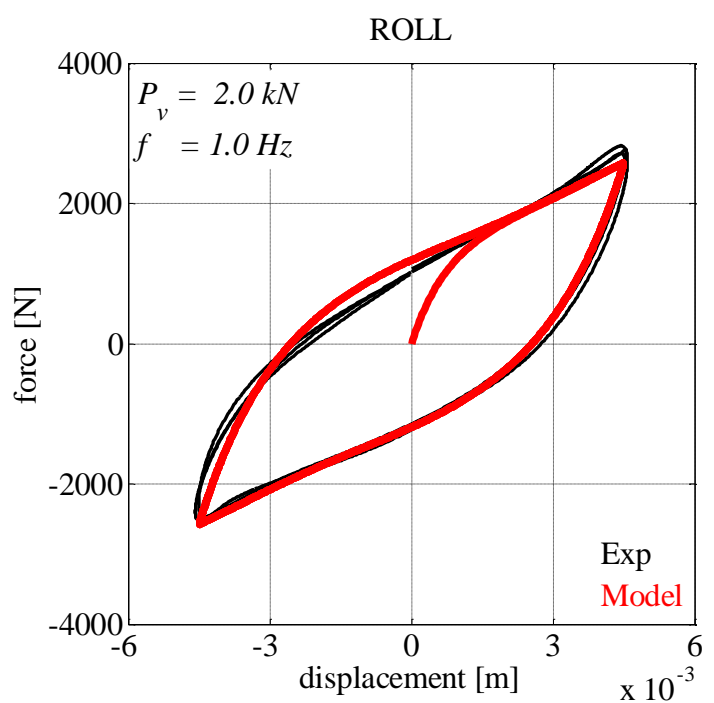

Figure 9. Experimental and parallel model loops for $A=0.005 \mathrm{~m}$.

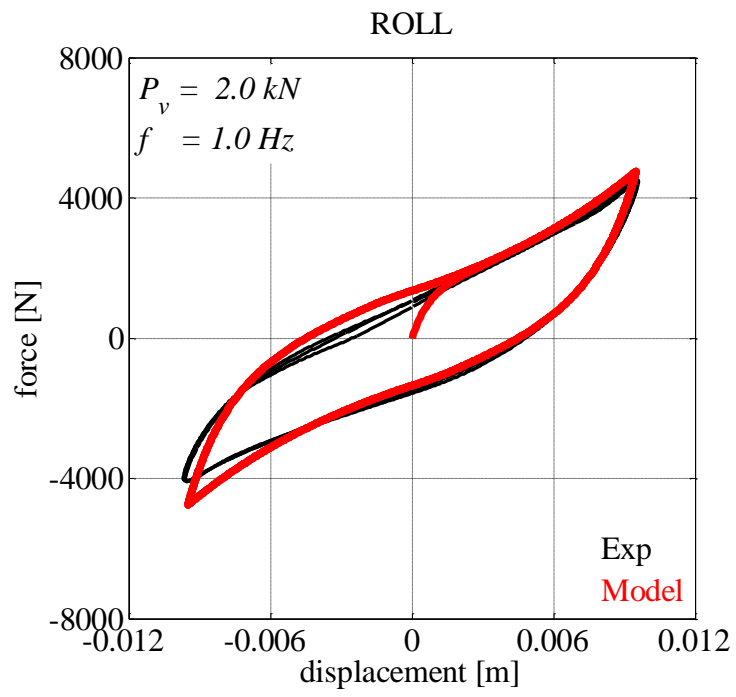

Figure 10. Experimental and parallel model loops for $A=0.01 \mathrm{~m}$.

As the same good agreement between the numerical and experimental responses has been obtained in Shear direction, these results are omitted for brevity. 
Proc. of the Fourth International Conference on Advances in Civil, Structural and Environmental Engineering - ACSEE 2016. Copyright (C) Institute of Research Engineers and Doctors. All rights reserved. ISBN: 978-1-63248-114-6 doi: 10.15224/ 978-1-63248-114-6-29

It is important to note that the proposed parallel model allows to capture the feature of the smooth transition of the hysteresis loops from the small to large horizontal displacements ranges by adopting only one set of five parameters, namely $k_{0}, k_{\infty}, c_{1}, c_{2}$, and $c_{3}$, determined from experimental loops with the largest amplitude (Fig. 11). To account for the effects of the vertical load, the model parameters have to be adjusted based on experimental results.

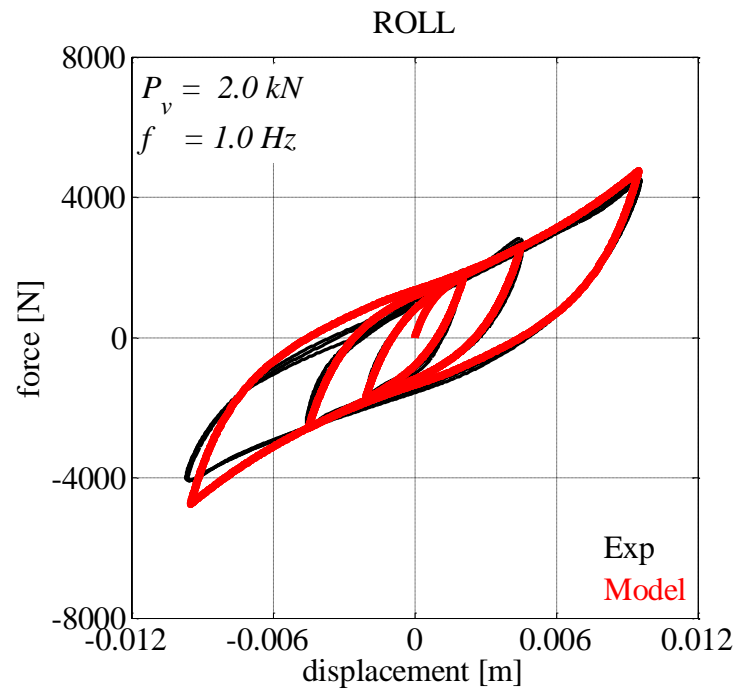

Figure 11. Hysteresis loops with different amplitudes simulated using only one set of five model parameters.

Furthermore, it is worth to point out that the proposed model allows to avoid the numerical solution of a first order nonlinear differential equation, for each time step of a nonlinear time history analysis, required by differential equation models such as the widely used Wen model [10].

\section{v. Conclusions}

In this work, the experimental tests performed on a HWRI have been presented in order to describe the dynamic behavior of the tested device in Roll and Shear directions. According to the experimental results, the selected HWRI exhibits symmetric softening force-displacement hysteresis loops within a small displacements range and a hardening stiffness at larger displacements.

A 1d parallel model has been proposed to predict the horizontal dynamic behavior of HWRIs at both small and large displacements: applying the parallel modeling concept to discretize an exponential function proposed by the authors to reproduce the variation of the horizontal tangent stiffness within the large displacements range, the force-displacement hysteresis loops have been simulated by putting in parallel one purely linear elastic element and a sufficiently high number of simple elastic-perfectly plastic elements and linear elastic gap elements.

Good agreement between the numerical and experimental results shows that the proposed parallel model can be an effective numerical tool to predict the horizontal dynamic behavior of HWRIs within the large displacements range. Making use of simple elastic-perfectly plastic elements and linear elastic gap elements, the presented parallel model, which is able to simulate hysteresis loops having different displacement amplitudes by adopting only one set of five model parameters determined from experimental loops with largest amplitude, is sufficiently versatile to be easily implemented in existing nonlinear finite element computer programs. Finally, compared to the widely used differential equation Wen model, the proposed one does not require the numerical solution of a first order nonlinear ordinary differential equation for each time step of a nonlinear time history analysis, thus reducing the computation effort.

\section{Acknowledgment}

The tested Helical Wire Rope Isolator was manufactured and provided by Powerflex S.r.l. (Limatola, Italy). The authors are grateful to this company and want to thank Prof. Filip C. Filippou for the possibility given to the first author to work on the Simulation of the Seismic Response of BaseIsolated Structures during his research period at the University of California at Berkeley.

\section{References}

[1] M. L. Tinker, and M. A. Cutchins, "' Damping phenomena in a wire rope vibration isolation system,', J. Sound Vib., 157(1), 7-18.

[2] G. F. Demetriades, M. C. Constantinou, and A. M. Reinhorn, 'Study of wire rope systems for seismic protection of equipment in buildings,', Eng. Struct., 15(5), 321-334.

[3] G. Serino, F. Bettinali, and G. Bonacina, 'Seismic base isolation of gas insulated electrical substations: comparison among different solutions," Proceedings of the 4th US Conference on Lifeline Earthquake Engineering 1995; San Francisco, California

[4] G. Serino, G. Bonacina, and F. Bettinali, 'Passive protection devices for high-voltage equipment: design procedures and performance evaluation," Proceedings of the 4th US Conference on Lifeline Earthquake Engineering 1995; San Francisco, California.

[5] M. Di Donna, and G. Serino, 'Base isolation of high-voltage equipment: comparison between two different solutions," Proceedings of the 7th International Conference on Probabilistic Methods Applied to Power Systems 2002; Napoli, Italy.

[6] S. Alessandri, R. Giannini, F. Paolacci, and M. Malena, 'Seismic retrofitting of an HV circuit breaker using base isolation with wire ropes. Part 1: preliminary tests and analyses,' Eng. Struct., 98, 251262.

[7] S. Alessandri, R. Giannini, F. Paolacci, M. Amoretti, and A. Freddo, 'Seismic retrofitting of an HV circuit breaker using base isolation with wire ropes. Part 2: shaking-table test validation,"' Eng. Struct., 98, 263-274.

[8] G. De Canio G, 'Marble devices for the base isolation of the two bronzes of Riace: a proposal for the David of Michelangelo," Proceedings of the 15th Word Conference on Earthquake Engineering 2012; Lisbon, Portugal.

[9] M. Spizzuoco, Q. Virginio, A. Calabrese, G. Serino, and C. Zambrano, "Study of wire rope devices for improving the recentering capability of base isolated buildings,' Struct. Control Health Monit., DOI: 10.1002/stc.1928.

[10] Y. K. Wen, 'Method for random vibration of hysteretic systems,' J. Eng. Mech. Div. - ASCE, 102, 249-263.

[11] S. Pagano, M. Russo, S. Strano, and M. Terzo, 'A mixed approach for the control of a testing equipment employed for earthquake isolation systems,'” J. Mech. Eng. Sci., 228(2), 246-261. 